\title{
INFLUENCIA DEL SISMO DE GOLFITO DEL 30 DE JULIO DE 2002 (Mw 6,2) SOBRE UNA PRUEBA DE BOMBEO EN EL ACUÍFERO CONFINADO DEL SITIO DE PRESA DEL P.H. PIRRÍS, COSTA RICA
}

\author{
INFLUENCE OF THE GOLFITO EARTHQUAKE OF 30 JULY 2002 (Mw 6,2) \\ OVER A PUMPING TEST IN THE CONFINED AQUIFER IN DAM SITE OF \\ PIRRIS HYDROELECTRIC PROJECT
}

\author{
Asdrúbal G. Vargas \\ Centro de Servicio Exploración Subterránea, Instituto Costarricense de \\ Electricidad (I.C.E.), Apdo. 10032-1000 San José Costa Rica. \\ avargass@ice.go.cr
}

(Recibido: 01/01/2015; aceptado: 01/06/2016)

\begin{abstract}
In order to determine the hydrogeological parameters of the fractured confined aquifer founded in the dam site of the Pirrís hydroelectric Power, a pumping test was carry out during 2560 minutes with an average flow rate of $1,9 \mathrm{l} / \mathrm{s}$, using a submersible pump of 5,6 kW. Approximately 420 minutes after the beginning of the pumping test a earthquake with a magnitude of $6.2 \mathrm{M}_{\mathrm{w}}$ with an epicenter near the town of Golfito (133,7 km far away), cause a significant change in the drawdowns values observed in four piezometers produced by the pumping in the well. After the event a recovery of the groundwater level in all piezometers were recorded, although the well continued with the extraction process. Using the recorded level and the time is possible the identification in a graph of five phases. Phase 1 corresponds to a drawdown of groundwater level caused by pumping with a 6-hour period; the second phase shows a sharp recovery as a result of the earthquake. The third phase is related to stabilization levels after the earthquake, however then a new level recovery phase occurs due to several aftershocks and finally another period of small drawdown is presented. After the test the data were reviewed to determine the effect of various parameters. For example partial penetration of the well, and the dip of the aquifer. From this preliminary analysis it was concluded that these factors did not influence the traditional method for the estimation of hydrodynamic parameters, therefore it was decided to analyze the data by the method of Theis and Moench for confined and fractured aquifers respectively.

Keywords: Golfito Earthquake, confined aquifer, pumping test, hydrodinamic parameters, fractured aquifer, hydrogeology.

RESUMEN: Con el fin de determinar los parámetros hidrogeológicos del acuífero fracturado de tipo confinado encontrado en el sitio de presa del Proyecto Hidroeléctrico Pirrís, se realizó una prueba de bombeo que se prolongó durante 2560 minutos, con un caudal promedio de 1,9 1/s, utilizando una bomba sumergible de 5,6 kW. Aproximadamente a los
\end{abstract}

VARGAS, A. G., 2016: Influencia del sismo de Golfito del 30 de julio de 2002 (Mw 6,2) sobre una prueba de bombeo en el acuífero confinado del sitio de presa del P.H. Pirrís, Costa Rica.- Rev. Geol. Amér. Central, 54: 143-153, DOI: 10.15517/rgac.v54i0.21151 
420 minutos de iniciada la prueba fue perturbada por un sismo con una magnitud de 6,2 Mw con epicentro a 133,7 km de distancia, ocasionando una importante modificación en el régimen de abatimientos impuesto por el bombeo en el pozo. Luego del evento se registró una recuperación del nivel piezométrico prácticamente en todos los piezómetros, aunque el pozo continuó con el proceso de extracción. Utilizando el gráfico de abatimiento registrado en el tiempo, se identificaron cinco fases. La fase 1 corresponde a un abatimiento del nivel piezométrico provocado por el bombeo con un período de 6 horas, la segunda fase muestra una recuperación brusca como resultado del sismo. La tercera fase se relaciona con una estabilización de los niveles después del sismo, no obstante seguidamente se presenta una nueva etapa de recuperación del nivel debido a varias réplicas sísmicas y finalmente se presenta una fase de abatimiento. Una vez finalizada la prueba, se procedió a la revisión de los datos de abatimiento para determinar el efecto de varios parámetros sobre el análisis del tipo de acuífero, debido a factores tales como: la penetración parcial del pozo y, el buzamiento del acuífero. De este análisis preliminar se dedujo que dichos factores no influyen sobre el método tradicional para obtener los parámetros hidrodinámicos, por consiguiente se decidió analizar la información por el método de Theis para acuíferos confinados y Moench para acuíferos fracturados.

Palabras clave: Terremoto Golfito, acuífero confinado, prueba bombeo, parámetros hidrodinámicos, acuífero fracturado, hidrogeología.

\section{INTRODUCCIÓN}

En el Sitio de Presa del proyecto hidroeléctrico Pirrís se identificó un acuífero de tipo confinado, cuya zona de recarga se localiza a unos $650 \mathrm{~m}$ aguas arriba del eje, dentro del área del embalse. Para conocer los parámetros hidrodinámicos se procedió a ejecutar y analizar una prueba de en el acuífero en mención con un pozo de bombeo ubicado a unos $60 \mathrm{~m}$ aguas arriba del eje de la presa (Fig. 1). La existencia de este acuífero, se detectó por primera vez en la margen izquierda, mediante la perforación PHP 52 SP, según se describe en el informe geológico geotécnico de factibilidad del sitio de presa No.2 presentado por la Dirección de Ingeniería Civil en 1997. Este informe contiene varias características descriptivas del acuífero artesiano, basadas en la información de la perforación PHP 52 SP. Posteriormente, otras perforaciones reafirmaron la extensión areal del acuífero y aportaron información que llevó a la identificación precisa del mismo y localización de la zona de recarga, que ha sido comprobada mediante estudios adicionales de geología, hidrogeología y geofísica. En el 2001, Chaves, Vargas \& Chávez presentan una caracterización hidrogeológica del sitio de presa, que incluye la versión actualizada del modelo conceptual del acuífero artesiano, fundamentado en la información recabada por las perforaciones, trabajo geológico de campo, geofísica e hidrogeoquímica.
La prueba de bombeo tuvo como objetivo determinar la conductividad hidráulica y el coeficiente de almacenamiento del acuífero confinado que se encuentra debajo del sitio de presa Pirrís. Así mismo, era de interés estimar el radio de influencia del cono de abatimiento del pozo de bombeo. La ejecución de la prueba se realizó por medio de un pozo de bombeo y cuatro piezómetros de observación ubicados en el sitio de presa y hacia aguas abajo.

El pozo de bombeo se denominó PHP 1DT SP $\mathrm{y}$ fue perforado verticalmente en un diámetro de $25 \mathrm{~cm}$, usando una máquina de rotopercusión con martillo de botones en el fondo. Originalmente el pozo se programó para una profundidad de $75 \mathrm{~m}$, sin embargo, debido a la extrema dureza y abrasividad del material que constituye el acuífero (capa silícea), que provocó el desgaste total de una broca a razón de unos $2 \mathrm{~m}$ de perforación, se decidió finalizar el pozo a los $65,5 \mathrm{~m}$, lográndose penetrar solo 2,5m en el acuífero (espesor promedio $14 \mathrm{~m}$ ). El diseño geomecánico del pozo incluye un tubo de $15 \mathrm{~cm}$ de diámetro con una longitud de $63,5 \mathrm{~m}$, ranurado en los últimos $12 \mathrm{~m}$ con una línea de aire de $2,5 \mathrm{~cm}$ de diámetro ranurada en los últimos $9 \mathrm{~m}$.

Desde el pozo de bombeo se extrae un caudal constante de agua impulsado por una bomba eléctrica, generando una depresión o cono de abatimiento del nivel piezométrico, que conforme transcurre el tiempose manifiesta en un descenso de los 


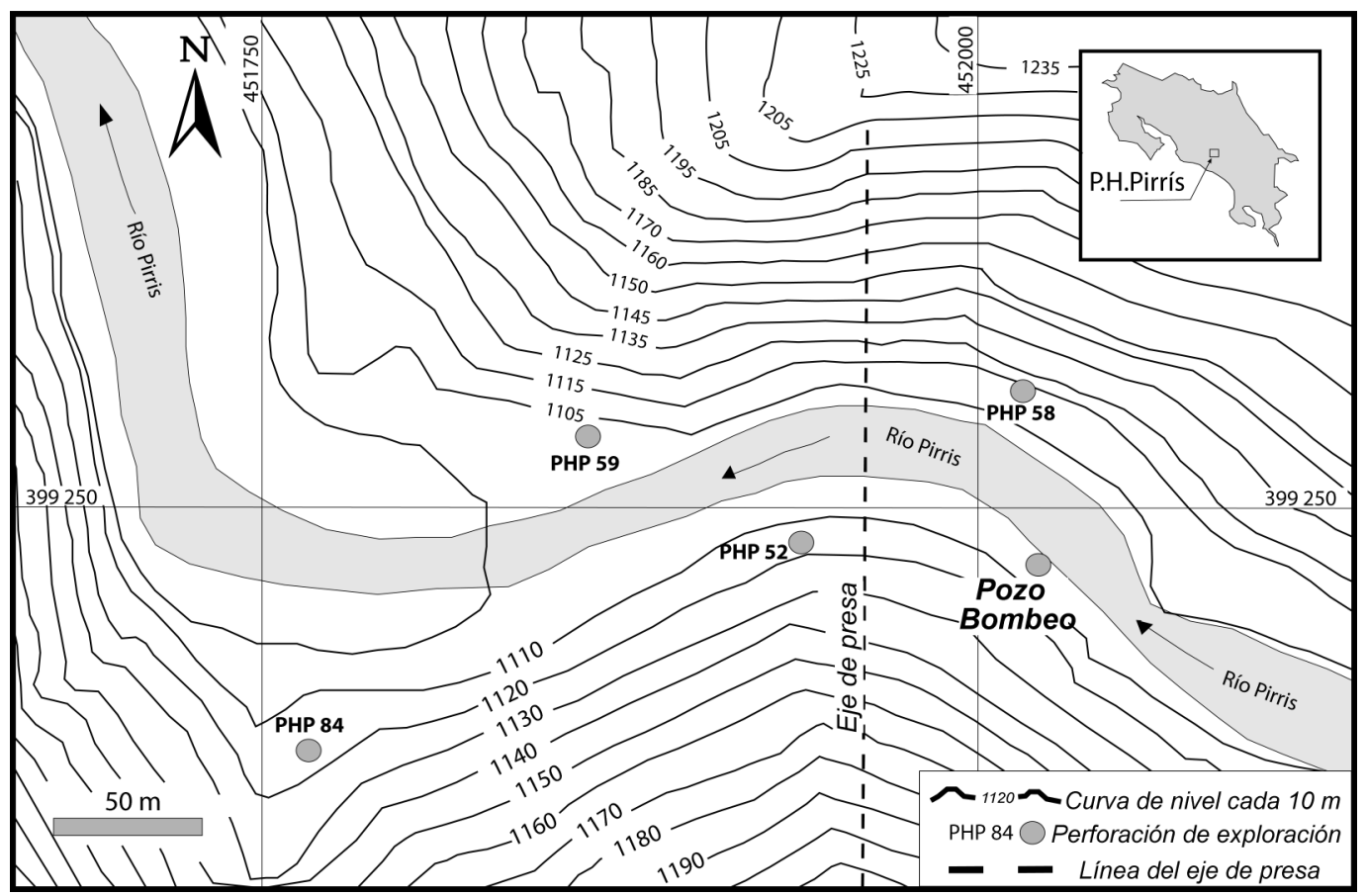

Fig. 1: Ubicación del pozo de bombeo y de los piezómetros.

niveles de agua de los piezómetros de observación (PHP: 52, 58, 59 y 84 SP). Por el carácter surgente de 3 de las perforaciones (PHP: 52, 58 y 59 SP), las mediciones se efectuaron directamente sobre una manguera plástica transparente $(12,5 \mathrm{~mm}$ de diámetro) que sirvieron como prolongaciones de los piezómetros. En la cuarta perforación (PHP $84 \mathrm{SP}$ ), las lecturas se tomaron por medio de una sonda con sensor electrosonoro. Una vez recolectados los datos, éstos se analizaron mediante diferentes métodos para determinar los parámetros hidrodinámicos, optando al final por el método de Theis, puesto que se ajusta mejor a las condiciones particulares y comportamiento mostrado por el acuífero en estudio.

El área de estudio se caracteriza por ser una depresión morfológica en forma de $\mathrm{V}$ con laderas que tienen pendientes que varían de $40^{\circ}$ a $50^{\circ}$, y una zona de erosión fluvial con pendientes menores. Longitudinalmente el río presenta varias inflexiones y recibe varios afluentes menores. Está caracterizado por una secuencia de rocas sedimentarias compuestas por lutitas, areniscas y brechas con cemento carbonatado, asociadas a la Formación Térraba. Según (DIG, 1997) las rocas sedimentarias se encuentran estratificadas y muestran un buzamiento general de 15 a $20^{\circ}$ con una dirección de buzamiento NW. En el sitio de presa, el macizo rocoso está afectado por tres familias de fracturas tectónicas, con rumbos dominantes N-S. Por lo general se trata de fracturas cerradas y en su mayoría rugosas onduladas y rugosas escalonadas, en muchos casos rellenas de calcita (Chaves \& Zumbado, 1999). Es probable que sean de carácter compresivo, influenciadas por un eje de esfuerzos máximo orientado al 034-214 (López, 1998). Por otra parte, existe una fuerte unión entre los estratos sedimentarios, transmitiendo al medio rocoso una mayor calidad física y reducción de la permeabilidad (Chaves \& Zumbado, 1999).

Estructuralmente, el sitio de presa está limitado por las fallas de rumbo denominadas Zapote y DS 2. La Falla Zapote es la más importante y se ubica a unos $300 \mathrm{~m}$ al sur del sitio donde se realizó la prueba de bombeo (Fig. 2). La misma pone en contacto directo las rocas sedimentarias con vulcanitas pertenecientes al Complejo 


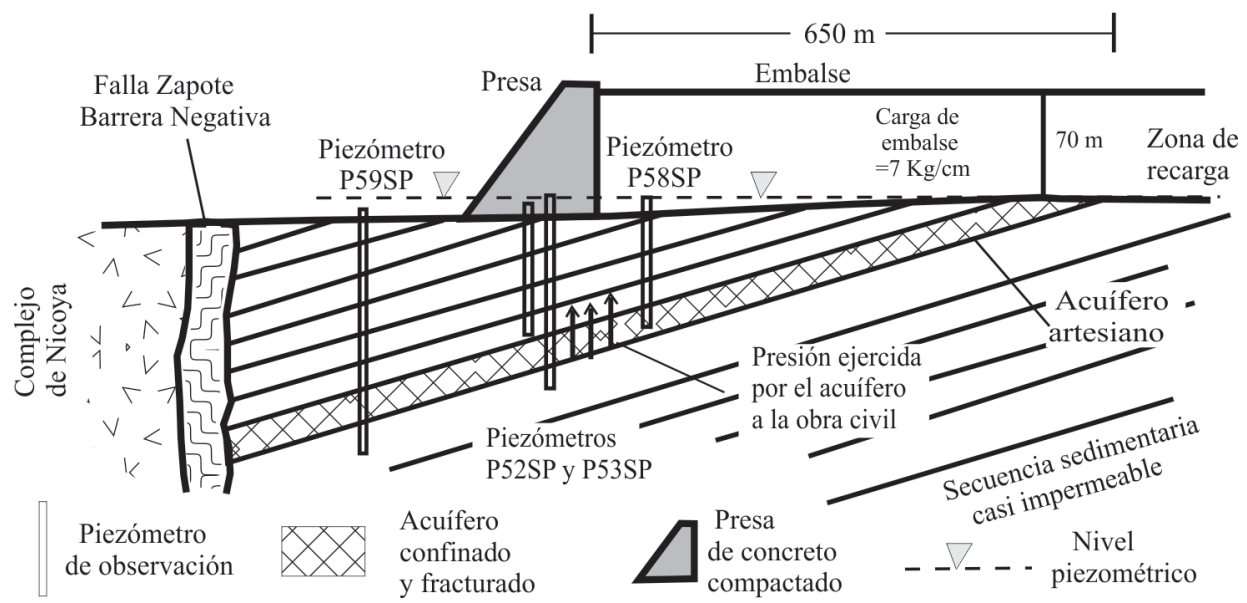

Fig. 2: Perfil esquemático del acuífero fracturado de tipo confinado.

de Nicoya, que incluyen lavas en almohadillas, gabros y microgabros, doleritas y sedimentos pelágicos (DIG, 1997).

\section{CONDICIONES HIDROGEOLÓGICAS}

La información suministrada por las diferentes campañas de perforación en el sitio de presa, confirman la existencia de un acuífero libre y uno confinado de tipo artesiano en rocas sedimentarias. En ambos casos, la matriz impermeable no favorece el paso del agua. Este ocurre a través de fisuras, existiendo un notable control de las estructuras tales como fallas y zonas de intenso fracturamiento, especialmente en el acuífero libre (Fig. 3). Aguas abajo del sitio de presa se encuentra un acuífero libre en los basaltos del Complejo de Nicoya, sin embargo, existen diferencias del nivel freático entre el acuífero en rocas sedimentarias y los basaltos muy notables.

La existencia de este acuífero confinado de tipo fracturado se detectó por primera vez mediante la perforación PHP 52 SP. Posteriormente, se confirmó durante la ejecución de las perforaciones PHP 53, 58, 59 y 63 SP y finalmente con las perforaciones PHP: 82, 84, 85 y 1 DT SP (Chávez et al, 2001). Todas estas perforaciones, con excepción de la PHP 63 SP, muestran artesianismo.
En la perforación PHP 53 SP, el confinamiento se presentó casi $40 \mathrm{~m}$ antes de alcanzar la capa silicificada, sin embargo, la surgencia ocurrió una vez que se penetró dicha capa. Por su parte, las perforaciones PHP: 52 y 63 SP no interceptaron la capa, pero experimentaron el fenómeno de artesianismo. Según los registros y gráficos de perforación, en las perforaciones PHP: 58 y 59, se evidencia una excelente correlación entre la manifestación del artesianismo y la presencia de un nivel de roca de aproximadamente $13 \mathrm{~m}$ de espesor con propiedades particulares. Este es posible localizarlo a unos $85 \mathrm{~m}$ bajo el sitio de presa y sobresale por su coloración clara, a diferencia del color oscuro de las rocas carbonatadas.

De acuerdo con los núcleos de perforación, la disposición espacial de la capa silicificada es concordante con la estratificación de la secuencia sedimentaria. Según se interpreta, se trata de un nivel intercalado de la secuencia, constituido por paquetes métricos de granulometría gruesa (ruditas y areniscas), que originalmente poseían una permeabilidad primaria importante. Esta condición favoreció la penetración y difusión de fluidos hidrotermalizados, que ascendieron a través de fallas desde cuerpos intrusivos en estado de diferenciación magmática. Dichos fluidos provocaron una alteración hidrotermal de baja temperatura, manifestada por una intensa silicificación 


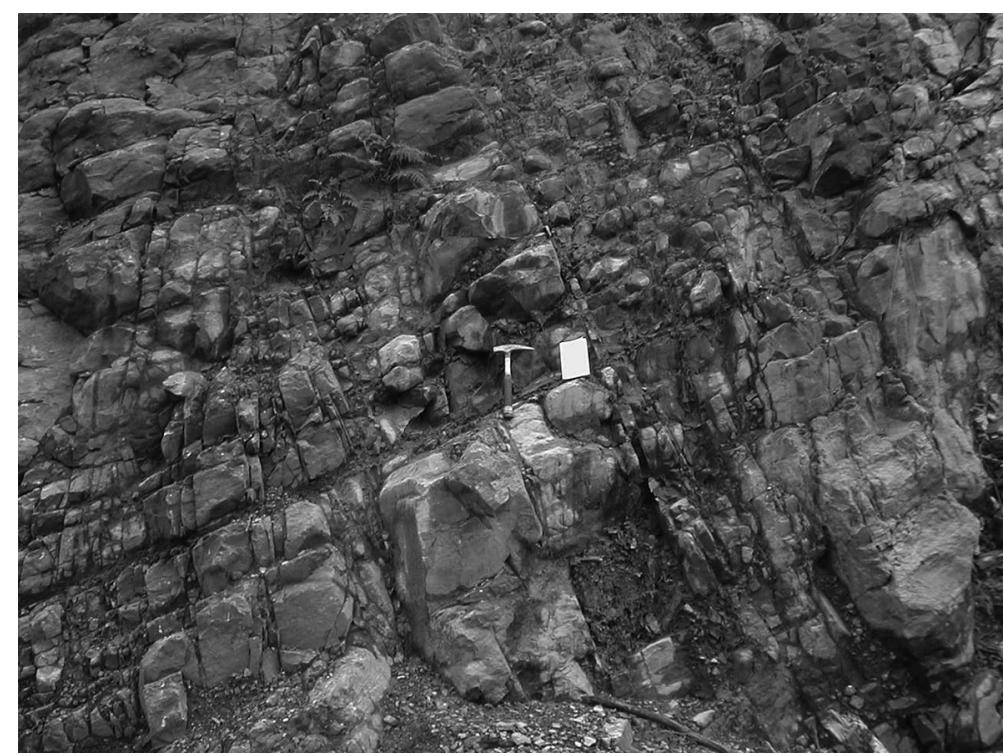

Fig. 3: Fracturas en las rocas sedimentarias.

en los intersticios, que transmitió a los niveles de roca afectados, cambios de coloración y endurecimiento anormal. Posteriormente, la silicificación indujo a un comportamiento mecánico diferente ante los esfuerzos tectónicos, traducido en un mayor nivel de deformación frágil, en comparación con el resto de la secuencia sedimentaria. De esta manera, la capa silicificada se fracturó más intensamente, propiciando una permeabilidad secundaria que dió origen al acuífero. El confinamiento del mismo, no obedece a un nivel sello en especial. Este se debe a la misma secuencia sedimentaria sobreyacente, cuyas características de impermeabilidad son deducibles de los núcleos y pruebas de agua de las perforaciones descritas (Chaves et al., 2001).

$\mathrm{El}$ artesianismo en las perforaciones PHP 52 y 63 SP y en la PHP 53 SP, antes de interceptar la capa silícea, se relacionan con la existencia de zonas fracturadas, algunas con evidencias de fallamiento. Es probable que durante el proceso de perforación y realización de pruebas de agua, estas zonas fracturadas hayan sufrido perturbaciones de carácter tensional, que facilitaron el ascenso del agua bajo presión, hacia las perforaciones. El comportamiento diferente de la perforación
PHP 59 SP, puede corresponder con una baja densidad de fracturas en la capa silícea, presente a nivel local y que no favorece una buena comunicación hidráulica.

Con respecto a la zona de recarga y descarga del acuífero confinado y tomando como base la superficie piezométrica y la proyección del buzamiento de la capa silicificada, se determinó que la zona de recarga se ubica a unos $650 \mathrm{~m}$ aguas arriba del eje de presa. Este sitio coincide con amplios afloramientos de roca silicificada, expuestos a lo largo de $360 \mathrm{~m}$ de cauce del río, que muestra características físicas y composicionales similares a la encontrada en las perforaciones. De igual manera, concuerdan en espesor puesto que mediante cálculos simples se estimó que no sobrepasa los $15 \mathrm{~m}$.

La cercanía de la zona de recarga y el hecho de que el río alimenta el acuífero, parece confirmarse al tomar en cuenta los registros de presiones. Se identificó como la carga artesiana variaba cuando se presentaba un período de lluvias intensas, que provocaba incrementos en el caudal del río. Asimismo, se demuestra desde un punto de vista físico, que las perforaciones captan un mismo acuífero. 


\section{PRUEBA DE BOMBEO}

\section{Diseño}

Como parte del diseño de la prueba fue necesario perforar un pozo vertical con una profundidad de 65,5 m. Como parte del encamizado del pozo se utilizó una tubería de plástico PVC de 15 $\mathrm{cm}$ de diámetro y se ranuraron los últimos $12 \mathrm{~m}$ para captar el acuífero confinado. Para medir el nivel del agua subterránea se instaló una línea de aire por medio de un tubo de plástico PVC de 2,5 $\mathrm{cm}$ de diámetro con un ranurado en los últimos $9 \mathrm{~m}$. Para extraer el agua del pozo se utilizó una bomba sumergible con una capacidad de 7,0 1/s y una potencia de 5,6 KW.

Los piezómetros utilizados en la prueba fueron perforados durante la etapa de factibilidad del proyecto con el fin de obtener núcleos de roca, medir las presiones y los correspondientes niveles del agua subterránea. Por lo tanto para esta ocasión se aprovechó la existencia de tales perforaciones para utilizarlos como sondeos de observación. Sin embargo, de manera previa se acondicionó el sitio de cada piezómetro (Fig. 4), por medio de una limpieza de la vegetación, la instalación de mangueras en los piezómetros con nivel surgente y la colocación de la instalación eléctrica provisional para dotar de iluminación los sitios durante las lecturas en la fase nocturna de la prueba.

Con varias semanas de anticipación, se realizaron varias pruebas de bombeo con duraciones de 4 a 6 horas, mediante los cuales se estableció que el caudal adecuado de bombeo debería ser cercano a los 20 1/s. La prueba de bombeo se realizó por medio de un pozo de bombeo denominado PHP 1 DT SP, ubicado en las coordenadas 399231,4 de latitud y 452019,3 de longitud con una elevación de la boca de 1106,7 m.s.n.m. Asi mismo se utilizaron 4 piezómetros de monitoreo según se indican el cuadro 1 .

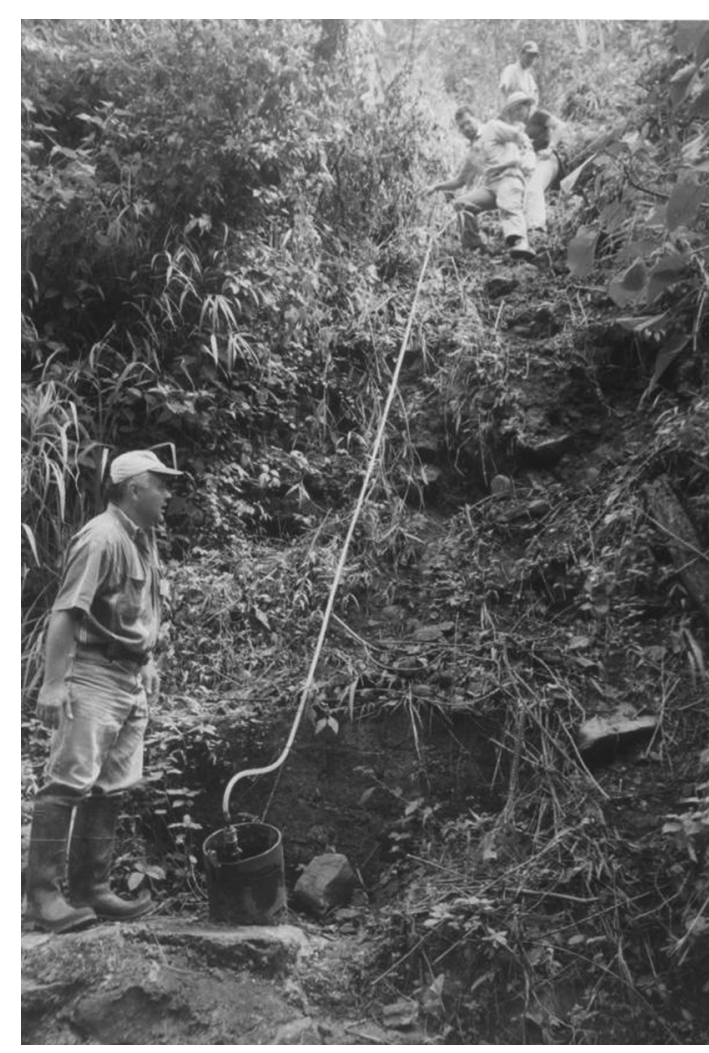

Fig. 4: Instalación de mangueras para lecturas en el piezómetro.

\section{Ejecución}

La prueba de bombeo se inició el día martes 30 de julio a las 11: 20 am y se prolongó hasta el día 01 de agosto, luego de 2560 minutos. El pozo se bombeó a un caudal de $1,85 \mathrm{l} / \mathrm{s}$ con variaciones de $0,15 \mathrm{l} / \mathrm{s}$, las cuales fueron relativamente bajas como para afectar el análisis por los métodos normales de caudal constante. En los piezómetros indicados en el cuadro 1 se midieron abatimientos según un esquema de mediciones temporales. Con este esquema se busca realizar mediciones del abatimiento al principio de la prueba 
Cuadro 1

Coordenadas de los piezómetros de monitoreo

\begin{tabular}{ccccc}
\hline Piezómetro & Latitud & Longitud & Elevación (m s.n.m) & $\begin{array}{c}\text { Distancia al pozo PHP } \\
\text { DT SP (m) }\end{array}$ \\
\hline PHP 52SP & 399238,620 & 451938,919 & 1118,302 & 81,80 \\
PHP 59SP & 399275,235 & 451864,315 & 1106,885 & 161,06 \\
PHP 84SP & 399166,891 & 451764,312 & 1111,590 & 263,02 \\
PHP 58SP & 399291,058 & 452015,042 & 1114,275 & 59,80 \\
\hline
\end{tabular}

con intervalos de tiempo relativamente cortos que aumentaron conforme se desarrolló la prueba. A los 420 minutos de iniciada la prueba $(6: 16 \mathrm{pm})$ ocurrió un sismo de magnitud Mw 6,2 con epicentro a $38 \mathrm{~km}$ de Puerto Armuelles en Panamá (Linkimer \& Barquero, 2003) a una distancia de $133,7 \mathrm{~km}$ de distancia de la prueba de bombeo, que causó un notorio proceso de recuperación del nivel piezométrico en todos los piezómetros a pesar de que el bombeo continuó.

\section{Resultados}

Utilizando el gráfico de abatimiento versus tiempo (Fig. 5), es posible identificar cinco fases. La fase 1 corresponde a un abatimiento del nivel piezométrico provocado por el bombeo con un período de 6 horas, la segunda fase muestra una recuperación brusca como resultado del sismo de Magnitud (Mw) 6,2, siendo mayor en el piezómetro PHP 59, el cual está a una distancia de 160 $\mathrm{m}$ del pozo de bombeo. Este proceso se debe a un efecto compresivo que cerró las fracturas que contenían agua e incrementó la presión hidráulica con la consecuente elevación de los niveles piezométricos. La tercera fase se relaciona con una estabilización de los niveles después del sismo, no obstante seguidamente se presenta una nueva etapa de recuperación del nivel debido a varias réplicas sísmicas y finalmente se presenta una fase de abatimiento.

Para la primera fase se observa que la respuesta del nivel piezométrico en los piezómetros PHP 52 SP y PHP 58 SP es muy pronunciada, alcanzado un abatimiento máximo de $0,80 \mathrm{~m}$, mientras que en los piezómetros PHP 59 SP y PHP 84 SP el abatimiento registrado es muy pequeño (menor de 0,07 m; Cuadro 2). Analizando los datos en detalle dentro de esta fase se observa un cambio de pendiente debido posiblemente a la presencia de una barrera negativa.

La distribución espacial del abatimiento muestra que el abatimiento más pronunciado se obtuvo cerca del pozo de bombeo y los piezómetros PHP 58 SP y PHP 52 SP, mientras que de menor valor hacia la perforación PHP 84 SP. Luego de que se disipó el efecto del temblor se estableció un pseudo equilibrio y con una leve recuperación.

\section{Interpretación}

Según Freeze \& Cherry (1979, pág. 343) los ensayos de bombeo permiten calcular valores de transmisividad y de coeficiente de almacenamiento representativos del sitio que se interpretan como un promedio sobre un volumen amplio de 


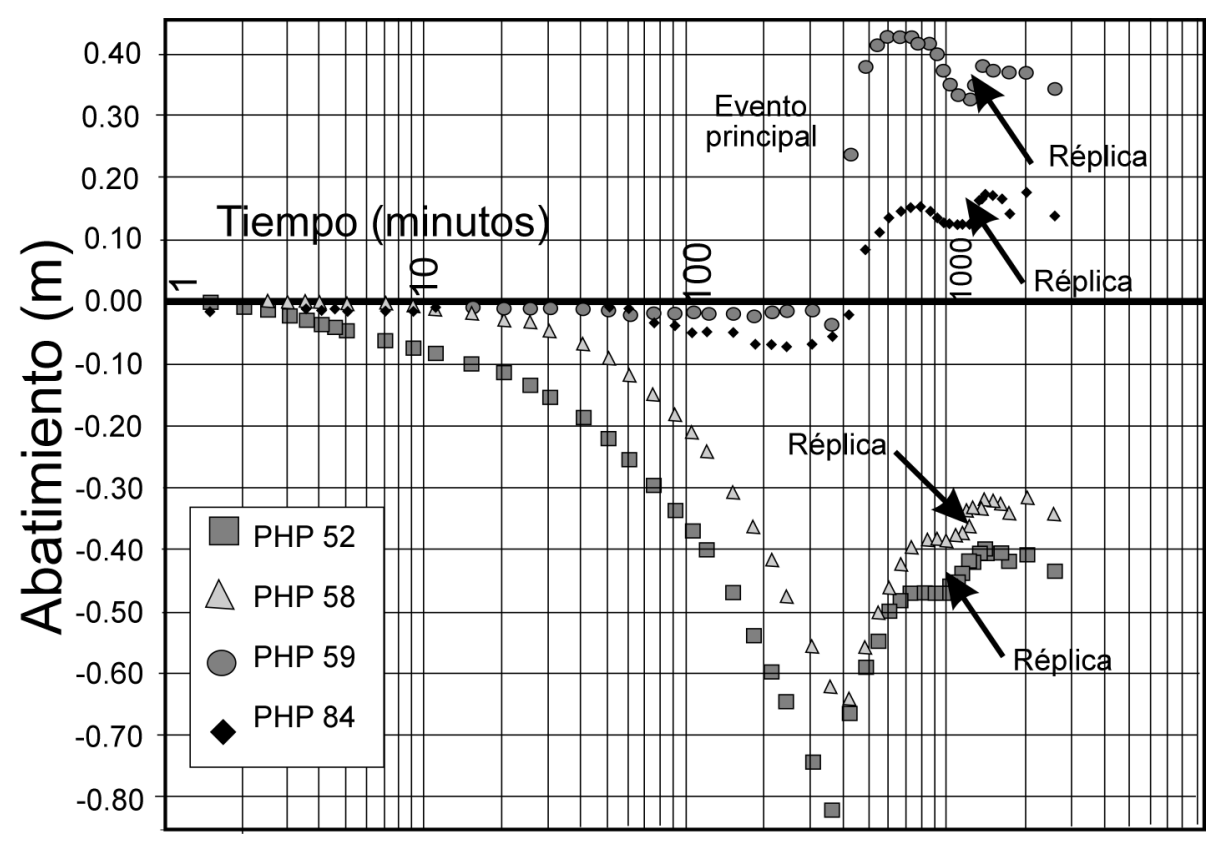

Fig. 5: Abatimiento registrado en los piezómetros durante la prueba de bombeo.

acuífero. Hay dos métodos clásicos de tipo gráfico para calcular los parámetros hidrodinámicos. El primero de ellos involucra el ajuste de los datos en un gráfico de tipo log-log (método de Theis) y la segunda interpretación se ejecuta con un gráfico semilogarítmico (método de Jacob). Kruseman \& De Rider (1994) presentan diferentes métodos para analizar pruebas de bombeo tomando en cuenta múltiples factores, entre ellos acuíferos fracturados. Así mismo Langgouth \& Voigt (1980) presentan los métodos clásicos de análisis de pruebas de bombeo e incluyen condiciones de frontera tanto positivas como negativas.

Según Kruseman \& De Ridder (1994) la interpretación de los datos de abatimiento para calcular las características hidrodinámicas de un acuífero implica identificar un sistema casi desconocido, ya que se desconoce en detalle las condiciones hidrogeológicas del acuífero. Así para identificar el sistema acuífero se debe comparar el comportamiento del abatimiento con modelos teóricos. Aquel modelo que se ajuste mejor con el comportamiento del sistema real es seleccionado para calcular las características hidráulicas. Por lo tanto la escogencia del modelo más adecuado para interpretar los datos de la prueba de bombeo se ha hecho comparando los datos con diferentes modelos teóricos.

Para la interpretación de los resultados se consideró que el acuífero es de tipo confinado debido a que en los piezómetros PHP 52 SP, PHP 58 SP y PHP 59 SP el nivel piezométrico es mayor que la elevación del techo del acuífero y es surgente lo que indica confinamiento del agua subterránea. Aunque algunas de estas condiciones son diferentes a las condiciones ideales que permiten analizar los datos de abatimiento por medio de los métodos tradicionales se utilizó el método tradicional de Theis para acuíferos confinados para estimar los parámetros hidrodinámicos, debido a que toma en cuenta que el acuífero la mayoría de condiciones, considerando que el acuífero es confinado y de una extensión areal infinita; el espesor del acuífero es uniforme sobre el área de influencia de la prueba; el pozo es bombeado a un caudal constante; antes del bombeo la superficie piezométrica es horizontal sobre el área que podría ser influenciada por la prueba de bombeo y el flujo de agua hacia el pozo es de tipo transiente. 
Cuadro 2

Cambio de abatimiento y recuperación en los niveles de agua

\begin{tabular}{|c|c|c|c|c|c|c|c|c|c|c|}
\hline Piezóm. & $\begin{array}{l}\text { Fase } 1 \\
(\mathrm{~m})\end{array}$ & $\begin{array}{c}\mathrm{t} \\
(\mathrm{min} .)\end{array}$ & $\begin{array}{c}\text { Fase } 2 \\
(\mathrm{~m})\end{array}$ & $\mathrm{t}(\min )$ & $\begin{array}{c}\text { Fase } 3 \\
(\mathrm{~m})\end{array}$ & $\mathrm{t}(\min )$ & $\begin{array}{c}\text { Fase } 4 \\
(\mathrm{~m})\end{array}$ & $\mathrm{t}(\min )$ & $\begin{array}{c}\text { Fase } \\
5 \\
(\mathrm{~m})\end{array}$ & $\mathrm{t}(\min )$ \\
\hline $\begin{array}{c}\text { PHP } 52 \\
\text { SP }\end{array}$ & 0,64 & $0-420(420)$ & 0,26 & $\begin{array}{c}420- \\
780(360)\end{array}$ & 0,0 & $\begin{array}{c}780- \\
1020(240)\end{array}$ & 0,065 & $\begin{array}{c}1080- \\
1380(300)\end{array}$ & 0,023 & $\begin{array}{c}1380- \\
2560(1180)\end{array}$ \\
\hline $\begin{array}{l}\text { PHP } 58 \\
\text { SP }\end{array}$ & 0,82 & $0-360(360)$ & 0,35 & $\begin{array}{c}360- \\
720(360)\end{array}$ & 0,0 & $\begin{array}{c}720-960 \\
(240)\end{array}$ & 0,07 & $\begin{array}{c}960- \\
1380(420)\end{array}$ & 0,03 & $\begin{array}{c}1380- \\
2560(1180)\end{array}$ \\
\hline $\begin{array}{l}\text { PHP } 59 \\
\text { SP }\end{array}$ & 0,03 & $0-360(360)$ & 0,40 & $\begin{array}{c}360- \\
600(240)\end{array}$ & 0,10 & $\begin{array}{c}600- \\
840(240)\end{array}$ & 0,05 & $\begin{array}{c}840- \\
1380(540)\end{array}$ & 0,03 & $\begin{array}{c}1380- \\
2560(1180)\end{array}$ \\
\hline $\begin{array}{c}\text { PHP } 84 \\
\text { SP }\end{array}$ & 0,07 & $0-300(300)$ & 0,23 & $\begin{array}{c}300- \\
720(420)\end{array}$ & 0,03 & $\begin{array}{c}720- \\
1080(360)\end{array}$ & 0,04 & $\begin{array}{c}1080- \\
1380(300)\end{array}$ & 0,03 & $\begin{array}{c}1380- \\
2560(1180)\end{array}$ \\
\hline
\end{tabular}

Aunque el pozo penetra parcialmente el acuífero confinado, se considera apropiado utilizar en la estimación de los parámetros $\mathrm{T}$ y $\mathrm{S}$, la información de abatimiento obtenida en todos los piezómetros, debido a que la distancia de observación supera el valor obtenido según la recomendación de Krusseman \& De Ridder (1994) de 1,5 b * $(\mathrm{Kv} / \mathrm{Kh}) 1 / 2$, que se estima en $21 \mathrm{~m}$. En este caso el espesor considerado es de $14 \mathrm{~m}$ y se ha utilizado una conductividad hidráulica vertical igual a la horizontal, debido al elevado grado de fracturamiento en varias direcciones que muestran las rocas que forman el acuífero.

Por lo tanto para la prueba de bombeo de Pirrís se ha analizado los datos de abatimiento por el método de Theis para acuífero confinado que se encuentra en las opciones de análisis de los datos del programa Aquifer Test, versión 3.0, desarrollado por Waterloo Hydrogeology. Con este programa el ajuste de los datos y el cálculo de la transmisividad, la conductividad hidráulica y el coeficiente de almacenamiento se realizan automáticamente, luego de introducir los valores de tiempo y la elevación del nivel del agua. Los valores de transmisividad, conductividad hidráulica y coeficiente de almacenamiento obtenidos del análisis de los datos de abatimiento para los piezómetros PHP 52 SP; PHP 58 SP y PHP 84 SP se muestran en el cuadro 3 .

Considerando que los datos previos a la influencia del sismo se ajustan relativamente bien con la curva patrón de Theis se decidió analizar los datos con este método mencionado. La transmisividad varía desde $67 \mathrm{~m}^{2} / \mathrm{d}$ hasta $2190 \mathrm{~m}^{2} / \mathrm{d}$. La conductividad hidráulica varía desde $26,9 \mathrm{~m} / \mathrm{d}$ hasta $880 \mathrm{~m} / \mathrm{d}$ y el coeficiente de almacenamiento se encuentra entre $3,8 \mathrm{E}^{-4}$ hasta $1,2 \mathrm{E}^{-3}$.

Por otro lado el fracturamiento tiene gran influencia sobre el flujo de un fluido dentro del macizo o la formación rocosa. Las ecuaciones convencionales de flujo hacia un pozo, desarrolladas inicialmente para acuíferos homogéneos, no describen adecuadamente el flujo en rocas fracturadas. Una excepción ocurre en rocas cristalinas de permeabilidad baja, si las fracturas son lo suficientemente numerosas y están distribuidas uniformemente a través de la roca; entonces el flujo de agua podría ocurrir solamente a través de las fracturas y podría ser similar al que ocurre en un acuífero homogéneo inconsolidado. No obstante el relleno de las fracturas puede jugar un papel muy importante en la permeabilidad según lo propone Moench (1984).

Los métodos de análisis están sustentados en la teoría de la doble porosidad desarrollado, es decir la formación rocosa está formada por dos medios: las fracturas y la porosidad de los bloques de la matriz, ambos con propiedades independientes. Por lo tanto coexisten dos porosidades y dos conductividades hidráulicas diferentes. Para el caso de la prueba de bombeo en el sitio de presa la permeabilidad generada por la porosidad primaria en la matriz es baja mientras que la permeabilidad generada por las fracturas es más elevada. Uno de 


\section{Cuadro 3}

Cambio de abatimiento y recuperación en los niveles de agua

\begin{tabular}{|c|c|c|c|c|c|c|}
\hline \multirow{2}{*}{ Piezómetro } & \multicolumn{3}{|c|}{ Método de Theis } & \multicolumn{3}{|c|}{ Método de Moench } \\
\hline & $\mathrm{T}(\mathrm{m} 2 / \mathrm{d})$ & $\mathrm{K}(\mathrm{m} / \mathrm{d})$ & $\mathrm{S}$ & $\mathrm{T}(\mathrm{m} 2 / \mathrm{d})$ & $\mathrm{K}(\mathrm{m} / \mathrm{d})$ & $\mathrm{S}(-)$ \\
\hline PHP 52 SP & 67,0 & 26,9 & 9,6 E-4 & 57,0 & 23,0 & $6,8 \mathrm{E}-4$ \\
\hline PHP 58 SP & 95,0 & 38,0 & $3,5 \mathrm{E}-4$ & 95,0 & 38,0 & $3,8 \mathrm{E}-4$ \\
\hline PHP 59 SP & 2190 & 880,0 & $1,2 \mathrm{E}-3$ & 2500,0 & 1000 & $1,2 \mathrm{E}-3$ \\
\hline PHP 84 SP & 110 & 8,9 & $5,5 \mathrm{E}-4$ & 73,0 & 28,0 & $5,2 \mathrm{E}-4$ \\
\hline
\end{tabular}

los métodos de análisis que se utilizó para medio fracturado fue el de Moench (1984). Este método genera una solución basada en las siguientes propiedades: acuífero confinado, anisotrópico y homogéneo de extensión infinita y de extensión uniforme a través del área de influencia del ensayo. Los resultados obtenidos para los parámetros hidrodinámicos se muestran en el cuadro 3 . Aplicando el método de Moench para acuíferos fracturados y utilizando los datos de abatimiento antes del sismo se obtuvo que la transmisividad varía desde $57,0 \mathrm{~m}^{2} / \mathrm{d}$ hasta $2500 \mathrm{~m}^{2} / \mathrm{d}$; la conductividad hidráulica varía desde $23 \mathrm{~m} / \mathrm{d}$ hasta 1000 $\mathrm{m} / \mathrm{d}$ y el coeficiente de almacenamiento varía en el mismo rango calculado por el método anterior.

\section{CONCLUSIONES}

Varios aspectos influyeron sobre la interpretación de los resultados de la prueba de bombeo, entre ellos la ocurrencia de un sismo que provocó la recuperación y oscilación de los niveles medidos en el campo. Por lo tanto se decidió calcular los valores de los parámetros hidrodinámicos considerando solo los datos previos al sismo. La evaluación de los datos de recuperación y oscilación provocados por el sismo no se logró llevar a cabo por el método convencional de recuperación de Theis puesto que el pozo de bombeo continuó extrayendo agua desde el acuífero, lo cual no está contemplado en el método tradicional.

Si bien el pozo de bombeo en el sitio de presa penetra parcialmente el acuífero confinado, la forma de la curva obtenida de los datos de campo se ajustan adecuadamente a la curva teórica de Theis, por lo tanto se ha considerado que no afecta el análisis realizado. Además aunque el acuífero presenta una inclinación (entre $15^{\circ}$ y $20^{\circ}$ ) se considera que no afectará el análisis de los datos de abatimiento puesto que no implica un cambio o disminución de la presión, ya que siempre tenderá a alcanzar la elevación de la zona de recarga.

Utilizando el gráfico de abatimiento versus tiempo, es posible identificar cinco fases. La fase 1 corresponde a un abatimiento del nivel piezométrico provocado por el bombeo con un período de 6 horas, la segunda fase muestra una recuperación brusca como resultado del sismo de 6,2 grados, siendo mayor en el piezómetro PHP 59, el cual está a una distancia de $160 \mathrm{~m}$ del pozo de bombeo. La tercera fase se relaciona con una estabilización de los niveles después del sismo, no obstante seguidamente se presenta una nueva etapa de recuperación del nivel debido a varias réplicas sísmicas y finalmente se presenta una fase de abatimiento.

El análisis de la curva de datos de abatimiento para los piezómetros PHP 52 SP y PHP 58 SP permite detectar que ocurre un cambio súbito de pendiente a los 50 y 40 minutos respectivamente. Este cambio se asocia con la probable presencia de una o varias barreras negativas, cuya distancia se calculó por medio de la expresión del radio de influencia del cono de abatimiento y se obtuvo una distancia de $98 \mathrm{~m}$ utilizando los valores de transmisividad y coeficiente de almacenamiento calculados con los datos del piezómetro PHP 52 SP. A esta distancia se conoce la presencia de una anomalía geofísica definida por Ávila (2001). 
Utilizando los valores de transmisividad y coeficiente de almacenamiento a partir de los datos del piezómetro PHP 58 SP se obtiene una distancia de $173 \mathrm{~m}$, de la barrera negativa, la cual parece tener relación con la zona de deformación de la Falla Zapote, ubicada a $300 \mathrm{~m}$ al SO del pozo de bombeo.

\section{REFERENCIAS}

ÁVILA, M., 2001: Estudio geofísico para el acuífero artesiano del sitio de presa.- P.H. PIRRIS.- 9 págs. I.C.E. C. S. Exploración Subterránea. San José [Informe interno].

CHÁVES, C. \& ZUMBADO, R., 1999: Informe preliminar de resultados e interpretaciones geológicas de las pruebas de impermeabilización hechas en el sitio de presa del P.H. Pirrís.- 29 págs. I.C.E. C. S. Exploración Subterránea. San José [Informe interno].

CHAVES, C., VARGAS, A. \& CHÁVEZ, J., 2001: Caracterización hidrogeológica del sitio de presa del proyecto hidroeléctrico Pirrís.- Tecnología ICE. 11 (1): 73-82.

DIRECCIÓN DE INGENIERÍA CIVIL，1997: Informe Geológico-Geotécnico de Factibilidad, Sitio de Presa No.2.- 51 págs.
I.C.E. Departamento de Ingeniería Civil. San José [Informe interno].

FREEZE, A. \& CHERRY, J., 1979: Groundwater604 págs. Prentice Hall. Nueva Jersey.

KRUSEMAN, G. P. \& De Ridder, N. A., 1994: Analysis and evaluation of pumping Test Data.- 377 págs. International Institute for Land Reclamation and Improvement. Holanda.

LANGGUTH, H. \& VOIGT, R., 1980: Hydrogeologische Methoden.- 486 págs. Springer Verlag. Berlin.

LINKIMER, L. \& BARQUERO, R., 2003: Sismos sentidos en Costa Rica durante el año 2002.12 Págs. RSN (UCR-ICE). San José.

LÓPEZ, A., RODRÍGUEZ, C., PIEDRA, J., ROJAS, L. \& CHÁVEZ, J. 1998: Informe geológico-geotécnico de factibilidad del sitio de presa No. 2.- 71 págs. Departamento de Ingeniería Geológica, Dirección de Ingeniería Civil. San José [Informe interno].

MOENCH, A., 1984: Double porosity models for a fisured groundwater reservoir with fracture skin.- Water Resources Research. 20(7): 831-846 
